\title{
Theory and design of compact hybrid microphone arrays on two-dimensional planes for three-dimensional soundfield analysis
}

\author{
Hanchi Chen, ${ }^{\text {a) }}$ Thushara D. Abhayapala, and Wen Zhang \\ Research School of Engineering, College of Engineering and Computer Science, \\ The Australian National University, Canberra, Australia
}

(Received 13 January 2015; revised 10 August 2015; accepted 16 October 2015; published online 17 November 2015)

\begin{abstract}
Soundfield analysis based on spherical harmonic decomposition has been widely used in various applications; however, a drawback is the three-dimensional geometry of the microphone arrays. In this paper, a method to design two-dimensional planar microphone arrays that are capable of capturing three-dimensional (3D) spatial soundfields is proposed. Through the utilization of both omnidirectional and first order microphones, the proposed microphone array is capable of measuring soundfield components that are undetectable to conventional planar omni-directional microphone arrays, thus providing the same functionality as 3D arrays designed for the same purpose. Simulations show that the accuracy of the planar microphone array is comparable to traditional spherical microphone arrays. Due to its compact shape, the proposed microphone array greatly increases the feasibility of 3D soundfield analysis techniques in real-world applications.
\end{abstract}

(C) 2015 Acoustical Society of America. [http://dx.doi.org/10.1121/1.4934953]

[KGS]

Pages: 3081-3092

\section{INTRODUCTION}

Three-dimensional (3D) soundfield decomposition based on spherical harmonic analysis has become a popular tool in the field of array signal processing. Applications of this technique can be found in both acoustic and radio frequency (RF) areas, such as spatial filtering and beamforming, ${ }^{1-3}$ room acoustic modeling, ${ }^{4,5}$ soundfield analysis, ${ }^{6,7}$ source localization, ${ }^{4,8,9}$ active noise control, ${ }^{10,11}$ and phase mode processing for antenna arrays. ${ }^{12}$ Spherical harmonic analysis based 3D soundfield decomposition reveals the underlying characteristics of the soundfield, thus allowing high accuracy manipulation and analysis of the soundfield. This requires a microphone array with $3 \mathrm{D}$ pick up capability to detect the soundfield. To the best of our knowledge, all of the previously developed microphone array structures designed for this purpose have a 3D geometry.

A well-studied type of such array configuration is the spherical array. Because its geometry coincides with the spherical harmonics, the sound signal captured by a spherical microphone array is well-suited for the spherical harmonic transform. ${ }^{6,7}$ The existing spherical arrays can be categorized into two major categories: the open sphere model and the rigid sphere model. Both models are widely used in research applications, such as room geometry inference ${ }^{13}$ and near field acoustic holography (NAH). ${ }^{14}$ An inherent drawback of the open sphere model is the numerical illconditioning problem, which is due to the nulls in spherical Bessel functions, thus the diameter of the microphone array has to be chosen carefully. It has been shown that such illconditioning problem can be overcome via methods such as

\footnotetext{
${ }^{\text {a)} E l e c t r o n i c ~ m a i l: ~ h a n c h i . c h e n @ a n u . e d u . a u ~}$
}

using concentric spheres ${ }^{15,16}$ or co-centered rigid/open spheres ${ }^{17}$ or by measuring the radial velocity. ${ }^{7}$

The placement of microphones on a spherical array has to follow a strict rule of orthogonality of the spherical harmonics ${ }^{18,19}$ which limits the flexibility of the array configuration. The spherical shape of the array also pose difficulties on implementation as well as practical usage.

Non-spherical microphone arrays have also been proposed for the purpose of 3D soundfield recording, such as the conical microphone array aperture proposed by Gulpta et $a l .{ }^{20}$ and the multiple circular microphone array proposed by Abhayapala et al. $^{21}$ These microphone arrays offer greater geometrical flexibility compared to spherical microphone arrays, thus allowing easier implementation of larger microphone arrays. However, these apertures still occupy a 3D space, which hinders the development of compact microphone arrays for practical applications.

On the other hand, microphone arrays featuring 2D geometry are easy to implement, yet existing 2D microphone arrays are incapable of capturing complete 3D soundfield information. Meyer et al. has shown that a 2D microphone array can be used to measure certain vertical component of a 3D soundfield. ${ }^{22}$ However, due to inherent properties of the spherical harmonics, some spherical harmonic modes are invisible to omni-directional pressure microphones on the $x-y$ plane, which explains why previously proposed 2D microphone arrays fail to extract full 3D soundfield information. Measurement of these soundfield components on the $x-y$ plane calls for additional types of sensors; no such technique has been proposed to our best knowledge.

First order microphones, such as differential microphones and cardioid microphones, are known to have the capability of detecting acoustic velocity in a certain direction. ${ }^{23}$ Kuntz et al. have shown that through using cardioid 
microphones pointed in the radial direction to replace omnidirectional microphones in a circular array, the numerical illconditioning problem can be solved for a 2D soundfield analysis system. ${ }^{24}$

In this work, we investigate using first order microphones to aid the detection of $3 \mathrm{D}$ soundfields and propose a new method for 3D soundfield recording using a 2D planar microphone array. In our approach, we use first order microphones in conjunction with omni-directional microphones to measure the "invisible" component of a 3D soundfield on the $x-y$ plane. Also we propose a method of using multiple co-centered circular arrays of omnidirectional/first order microphones to compute the soundfield coefficients associated with the spherical space enclosing the planar array aperture. We show that the proposed planar microphone array offers the same functionality as spherical/multiple circular arrays designed for soundfield analysis.

This paper is arranged as follows: Sec. II briefly reviews the soundfield analysis technique and derives the wave domain expression of soundfield measured by the general first order microphone. We show that the full 3D soundfield can be observed on a plane with the aid of first order microphones by exploiting a property of the associated Legendre functions. Section III introduces the co-centered hybrid circular microphone array for soundfield recording and shows how the soundfield coefficients can be calculated using the data measured by different components of the hybrid array. We also provide a step-by-step design procedure for determining parameters of an array based on system requirements. Section IV provides an analysis on the accuracy performance of the proposed array. Two primary causes of error are identified, and their impact on each soundfield coefficient is discussed. Section V gives a hypothetical design example of the proposed microphone array as well as an experimental microphone array built for validation of the theory. Detailed simulation results are provided for the hypothetical design example and the test results of the experimental array is compared with corresponding simulation results for performance evaluation.

\section{FIRST ORDER MICROPHONES FOR SOUNDFIELD ACQUISITION}

In this section, we derive the general velocity of the pressure field at a point along a direction and the wave domain expression of the received signal of a general first order microphone. We also show that the 3D soundfield coefficients can be divided into even and odd components; while the even modes can be measured by omnidirectional microphones, the odd components of the soundfield can be observed on a plane by using a recurrent relationship of associated Legendre functions.

\section{A. Spatial soundfield decomposition}

A soundfield within a source free region of space at a point $(r, \theta, \phi)$ with respect to an origin $O$ of the spherical coordinate system can be written as ${ }^{25}$

$$
P(r, \theta, \phi, k)=\sum_{n=0}^{\infty} \sum_{m=-n}^{n} C_{n m}(k) j_{n}(k r) Y_{n m}(\theta, \phi)
$$

where $C_{n m}(k)$ are soundfield coefficients, $k=2 \pi f / c$ is the wave number, $f$ is the frequency, $c$ is the speed of sound propagation, $j_{n}(k r)$ is the $n$th order spherical Bessel function of the first kind, $Y_{n m}(\theta, \phi)$ are the spherical harmonics, defined by

$$
Y_{n m}(\theta, \phi)=\mathcal{P}_{n|m|}(\cos \theta) E_{m}(\phi),
$$

where

$$
\mathcal{P}_{n|m|}(\cos \theta) \triangleq \sqrt{\frac{(2 n+1)}{4 \pi} \frac{(n-|m|) !}{(n+|m|) !}} P_{n|m|}(\cos \theta)
$$

and

$$
E_{m}(\phi) \triangleq(1 / \sqrt{2 \pi}) e^{j m \phi}
$$

are the normalized associated Legendre functions and normalized exponential functions, respectively; $P_{n|m|}(\cos \theta)$ are the associated Legendre functions. In literature, the representation Eq. (1) is referred to as spherical harmonic expansion, wave-domain representation, modal expansion, or multi-pole expansion of a wavefield.

For the case of soundfields, $P(r, \theta, \phi, k)$ is the pressure at a point as a function of frequency (wavenumber). The problem of soundfield acquisition is to extract the soundfield coefficients $C_{n m}(k)$ by sampling the soundfield over space and time. The specific problem we consider in this paper is to devise a method to extract 3D soundfield coefficients by sampling the field within a 2D plane.

\section{Wave domain expression of pressure gradient}

For reasons that will become clear later in the paper, we consider pressure gradient of a soundfield along the direction of $\theta$. That is, we consider either differential or velocity microphones placed in such a way that they measure pressure gradient in the direction of $\theta$ at a given point $(r, \theta, \phi)$.

We define the pressure gradient along the direction of $\theta$ at a point $(r, \theta, \phi)$ as

$$
P_{\theta}(r, \theta, \phi, k) \triangleq \frac{\partial P(r, \theta, \phi, k)}{\partial \theta} .
$$

By substituting Eq. (1) into Eq. (5) and taking the partial derivative with respect to $\theta$, the pressure gradient can be expressed as

$$
\begin{aligned}
P_{\theta}(r, \theta, \phi, k)= & -\sin \theta \sum_{n=0}^{\infty} \sum_{m=-n}^{n} C_{n m}(k) j_{n}(k r) \\
& \times \mathcal{P}_{n|m|}^{\prime}(\cos \theta) E_{m}(\phi),
\end{aligned}
$$

where

$$
\mathcal{P}_{n|m|}^{\prime}(u)=\frac{d \mathcal{P}_{n|m|}(u)}{d(u)}
$$


is the first order derivative of the normalized associated Legendre function.

\section{B. General expression for first order microphones}

The pick-up pattern of any first order microphone can be considered as a weighed sum of an omni-directional pattern and a differential pattern. Using $P(r, \theta, \phi, k)$ to represent the omnidirectional component of the measured sound pressure and $P_{\theta}(r, \theta, \phi, k)$ for the differential component in the $\theta$ direction at point $(r, \theta, \phi)$, the total sound pressure measured by an arbitrary first order microphone can be written as

$$
P_{c}(r, \theta, \phi, k) \triangleq \beta P(r, \theta, \phi, k)+(1-\beta) P_{\theta}(r, \theta, \phi, k),
$$

where $\beta$ is a weighing factor and has a range of $[0,1)$. When $\beta=0, P_{c}(r, \theta, \phi, k)$ contains only the differential pattern, which is considered as a special case of first order pick-up patterns. In this paper, differential microphones are regarded as one type of first order microphones; when $\beta$ $=0.5, P_{c}(r, \theta, \phi, k)$ becomes the pick-up pattern of a "standard" cardioid microphone. Substituting Eqs. (1) and (6) into Eq. (7) yields the wave domain representation of the signal received by a general first order microphone as

$$
\begin{aligned}
P_{c}(r, \theta, \phi, k)= & \sum_{n=0}^{\infty} \sum_{m=-n}^{n} C_{n m}(k) j_{n}(k r)\left(\beta \mathcal{P}_{n|m|}(\cos \theta)\right. \\
& \left.-(1-\beta) \sin \theta \mathcal{P}_{n|m|}^{\prime}(\cos \theta)\right) E_{m}(\phi)
\end{aligned}
$$

\section{Sampling on a plane}

Without loss of generality, let us place the coordinate system such that the plane of interest for sensor placement is the $x-y$ plane. In the spherical coordinate system, $\theta=\pi / 2$ (i.e., $\cos \theta=0$ ), for all points on the $x$-y plane. Thus the output of an omni-directional sensor place on the $x-y$ plane is

$$
P(r, \pi / 2, \phi, k)=\sum_{n=0}^{\infty} \sum_{m=-n}^{n} C_{n m}(k) j_{n}(k r) \mathcal{P}_{n|m|}(0) E_{m}(\phi)
$$

Observe that when $n+|m|$ is an odd integer, the value of $\mathcal{P}_{n|m|}(0)$ is equal to zero. ${ }^{21}$ Consequently, the spherical harmonics associated with these associated Ledengre functions are equal to zero. This property makes the odd mode spherical harmonics "invisible" on the $\theta=\pi / 2$ plane, which is why extraction of the complete $3 \mathrm{D}$ soundfield information cannot be done through sampling on a single plane using omni-directional microphones.

On the other hand,

$$
\mathcal{P}_{n|m|}^{\prime}\left(\cos \frac{\pi}{2}\right)=\left\{\begin{array}{l}
\text { a non-zero value, when } n+|m| \text { is an odd integer } \\
0, \text { when } n+|m| \text { is an even integer. }
\end{array}\right.
$$

Observe that the expression for the pressure gradient in Eq. (6) has the terms $\mathcal{P}_{n|m|}^{\prime}(\cdot)$. Hence the "odd" components of the pressure gradient along the direction of $\theta$ is non-zero on the $x-y$ plane. Thus the pressure gradient measurements contain odd $C_{n m}(k)$ (i.e., $n+|m|$ odd) coefficients. We use this property in this paper to propose a method to extract $3 \mathrm{D}$ soundfield components by sampling the field on the $x-y$ plane using differential (or first order) and omni-directional microphones together.

A recurrent relationship between the associated Legendre function and its first order derivative is given by ${ }^{26}$

$$
\left(x^{2}-1\right) \frac{d P_{n|m|}(x)}{d x}=n x P_{n|m|}(x)-(|m|+n) P_{(n-1),|m|}(x) \text {. }
$$

When $x=\cos (\pi / 2)=0,(10)$ reduces to

$$
P_{n|m|}^{\prime}(0)=(|m|+n) P_{(n-1),|m|}(0) .
$$

By taking the derivative of Eq. (3) and setting $\cos \theta=0$, expressing $P_{n|m|}^{\prime}(0)$ using Eq. (11) and expressing
$P_{(n-1),|m|}(0)$ with $\mathcal{P}_{n-1|m|}(0)$ using Eq. (3), we derive the following relationship for the normalised associate Legendre functions:

$$
\mathcal{P}_{n|m|}^{\prime}(0)=\sqrt{\frac{(2 n+1)\left(n^{2}-m^{2}\right)}{(2 n-1)}} \mathcal{P}_{(n-1)|m|}(0)
$$

By substituting Eq. (12) into Eqs. (6) and (8), we can write the output of the differential and general first order microphones placed at a point $(r, \pi / 2, \phi)$ on the $x$-y plane along the direction of $\theta$ (i.e., perpendicular to the $x-y$ plane) as

$$
\begin{aligned}
P_{\theta}\left(r, \frac{\pi}{2}, \phi, k\right)= & -\sum_{n=0}^{\infty} \sum_{m=-n}^{n} C_{n m}(k) j_{n}(k r) \\
& \times \sqrt{\frac{(2 n+1)\left(n^{2}-m^{2}\right)}{(2 n-1)}} \\
& \times \mathcal{P}_{(n-1)|m|}(0) E_{m}(\phi)
\end{aligned}
$$

and 


$$
P_{c}\left(r, \frac{\pi}{2}, \phi, k\right)=\sum_{n=0}^{\infty} \sum_{m=-n}^{n} C_{n m}(k) j_{n}(k r)\left(\beta \mathcal{P}_{n|m|}(0)-(1-\beta) \sqrt{\frac{(2 n+1)\left(n^{2}-m^{2}\right)}{(2 n-1)}} \mathcal{P}_{(n-1)|m|}(0)\right) E_{m}(\phi),
$$

respectively.

$$
P\left(R_{q}, \frac{\pi}{2}, \phi, k\right) \approx \sum_{n=0}^{N} \sum_{m=-n}^{n} C_{n m}(k) j_{n}\left(k R_{q}\right) \mathcal{P}_{n|m|}(0) E_{m}(\phi),
$$

\section{ARRAY CONFIGURATION}

In this section, we outline possible geometric configurations of first order and omni-directional sensors on the $x-y$ plane to extract both the even and odd spherical harmony components of the soundfield.

\section{A. Calculation of harmonic coefficients}

\section{Even coefficients: Omni-array}

Consider a circle placed on the $x-y$ plane such that an arbitrary point on the circle is given by $\left(R_{q}, \pi / 2, \phi\right)$. Then the output of a omni-directional microphone on the circle at $\left(R_{q}, \pi / 2, \phi\right)$ is given by

$$
P\left(R_{q}, \frac{\pi}{2}, \phi, k\right)=\sum_{n=0}^{\infty} \sum_{m=-n}^{n} C_{n m}(k) j_{n}\left(k R_{q}\right) \mathcal{P}_{n|m|}(0) E_{m}(\phi) .
$$

Because soundfields over a spherical region of finite radius are mode limited, ${ }^{25}$ the infinite summation on right hand side of Eq. (15) can be approximated by a finite sum, where $N$ denotes the maximum harmonic order at the array's radius $R_{q}$ and the highest operating frequency. ${ }^{27}$ Multiplying both sides of Eq. (16) by $E_{m}(-\phi)$ and integrating with respect to $\phi$ over $[0,2 \pi)$ yields the total sound pressure received by the ring, as

$$
\begin{aligned}
\alpha_{m}\left(R_{q}, k\right) & \triangleq \int_{0}^{2 \pi} P\left(R_{q}, \pi / 2, \phi, k\right) E_{m}(-\phi) d \phi \\
& =\sum_{n=|m|}^{N} C_{n m}(k) j_{n}\left(k R_{q}\right) \mathcal{P}_{n|m|}(0) .
\end{aligned}
$$

Note that only the even mode harmonics are present in Eq. (18) because $\mathcal{P}_{n|m|}(0)=0$ for $n+|m|$ odd. Let there be a total of $Q$ circles placed at different radii but all on the $\theta$ $=\pi / 2$ plane $(x-y$ plane $)$. Thus for $q=1, \ldots, Q$, the relationship between the even mode soundfield coefficients of mode $m$ and the azimuth sound pressure harmonics $\alpha_{m}\left(R_{q}, k\right)$ on each circle can be expressed as

$$
\alpha_{m}(k)=\boldsymbol{U}_{m}(k) \boldsymbol{C}_{m}^{\mathrm{even}}(k),
$$

where $\alpha_{m}(k)=\left[\alpha_{m}\left(R_{1}, k\right), \alpha_{m}\left(R_{2}, k\right), \ldots, \alpha_{m}\left(R_{Q}, k\right)\right]^{T}$,

$$
\boldsymbol{C}_{m}^{\mathrm{even}}(k)=\left\{\begin{array}{l}
{\left[C_{m m}(k), C_{(m+2) m}(k), \ldots, C_{N m}(k)\right]^{T}, \quad \text { if } m \text { and } N \text { are both even/odd }} \\
{\left[C_{m m}(k), C_{(m+2) m}(k), \ldots, C_{(N-1) m}(k)\right]^{T}, \text { otherwise }}
\end{array}\right.
$$

is the vector of the even mode coefficients of mode $m$ and

$$
\boldsymbol{U}_{m}(k)=\left[\begin{array}{cccc}
j_{m}\left(k R_{1}\right) \mathcal{P}_{m|m|}(0) & j_{m+2}\left(k R_{1}\right) \mathcal{P}_{(m+2)|m|}(0) & \cdots & j_{N}\left(k R_{1}\right) \mathcal{P}_{N|m|}(0) \\
j_{m}\left(k R_{2}\right) \mathcal{P}_{m|m|}(0) & j_{m+2}\left(k R_{1}\right) \mathcal{P}_{(m+2)|m|}(0) & \cdots & j_{N}\left(k R_{2}\right) \mathcal{P}_{N|m|}(0) \\
\vdots & \vdots & \vdots & \vdots \\
j_{m}\left(k R_{Q}\right) \mathcal{P}_{m|m|}(0) & j_{m+2}\left(k R_{Q}\right) \mathcal{P}_{(m+2)|m|}(0) & \cdots & j_{N}\left(k R_{Q}\right) \mathcal{P}_{N|m|}(0)
\end{array}\right],
$$

for the case with both $N$ and $m$ are either odd or even [otherwise replace $N$ in Eq. (21) by $N-1]$.

We can estimate the even mode coefficients from Eq. (19), provided $\boldsymbol{U}_{m}(k)$ is not singular, as

$$
\boldsymbol{C}_{m}^{\mathrm{even}}(k)=\boldsymbol{U}_{m}^{\dagger}(k) \alpha_{m}(k),
$$

where $\boldsymbol{U}_{m}^{\dagger}=\left(\boldsymbol{U}_{m}^{T} \boldsymbol{U}_{m}\right)^{-1} \boldsymbol{U}_{m}^{T}$ is the pseudo inverse of $\boldsymbol{U}_{m}$.

Note that the calculation of even harmonic coefficients are similar to the work reported in Ref. 21. However, we show in Sec. III A 2 how to extract odd harmonic coefficients by placing the differential microphones on the $x-y$ plane, which is a method not reported elsewhere to the best of our knowledge.

\section{Odd coefficients: Differential microphone array}

Consider a circular array of differential microphones with radius $R_{q}$ placed on the $x-y$ plane with all differential microphones pointed perpendicular to the $x-y$ plane (i.e., $\theta$ 
$=\pi / 2$ plane). Then the output of a differential microphone on the circle at $\left(R_{q}, \pi / 2, \phi\right)$ is given by Eq. (13). Using the properties of the spherical Bessel functions, we can show that the infinite summation of Eq. (13) can be truncated to a finite number [similar to the case of Eq. (16)]. The resulting equation is given in the following text:

$$
P_{\theta}\left(R_{q}, \frac{\pi}{2}, \phi, k\right)=-\sum_{n=0}^{N} \sum_{m=-n}^{n} C_{n m}(k) j_{n}\left(k R_{q}\right) \sqrt{\frac{(2 n+1)\left(n^{2}-m^{2}\right)}{(2 n-1)}} \mathcal{P}_{(n-1)|m|}(0) E_{m}(\phi)
$$

By multiplying both sides of Eq. (23) by $E_{m}(-\phi)$ and integrating with respect to $\phi$ over $[0,2 \pi)$, we obtain the response of the differential microphone array, named as azimuth pressure gradient harmonics

$$
\begin{aligned}
\alpha_{m}^{(\mathrm{d})}\left(R_{q}, k\right) \triangleq & \int_{0}^{2 \pi} P_{\theta}\left(R_{q}, \frac{\pi}{2}, \phi, k\right) E_{m}(-\phi) d \phi \\
= & -\sum_{n=|m|}^{N} C_{n m}(k) j_{n}\left(k R_{q}\right) \sqrt{\frac{(2 n+1)\left(n^{2}-m^{2}\right)}{(2 n-1)}} \\
& \times \mathcal{P}_{(n-1)|m|}(0)
\end{aligned}
$$

Note that only the odd mode harmonics are present in Eq. (25) because $\mathcal{P}_{(n-1)|m|}(0)=0$ for $n+|m|$ even.

By evaluating Eq. (25) for $q=1, \ldots, Q$, the relationship between the odd soundfield coefficients of mode $m$ and $\alpha_{m}^{(\mathrm{d})}\left(R_{q}, k\right)$ on each circle can be expressed as a matrix equation:

$$
\alpha_{m}^{(\mathrm{d})}(k)=\boldsymbol{V}_{m}(k) \boldsymbol{C}_{m}^{\mathrm{odd}}(k)
$$

where $\alpha_{m}^{(\mathrm{d})}(k)=\left[\alpha_{m}^{(\mathrm{d})}\left(R_{1}, k\right), \alpha_{m}^{(\mathrm{d})}\left(R_{2}, k\right), \ldots, \alpha_{m}^{(\mathrm{d})}\left(R_{Q}, k\right)\right]^{T}$,

$$
\boldsymbol{C}_{m}^{\text {odd }}(k)=\left\{\begin{array}{l}
{\left[C_{(m+1) m}(k), C_{(m+3) m}(k), \ldots, C_{(N-1) m}(k)\right]^{T}, \text { if } m \text { and } N \text { are both even } / \text { odd }} \\
{\left[C_{(m+1) m}(k), C_{(m+3) m}(k), \ldots, C_{N m}(k)\right]^{T}, \quad \text { otherwise }}
\end{array}\right.
$$

and

$$
\boldsymbol{V}_{m}(k)=\left[\begin{array}{cccc}
V_{(m+1)|m|}^{(1)} & V_{(m+3)|m|}^{(1)} & \ldots & V_{(N-1)|m|}^{(1)} \\
V_{(m+1)|m|}^{(2)} & V_{(m+3)|m|}^{(2)} & \ldots & V_{(N-1)|m|}^{(2)} \\
\vdots & \vdots & \ddots & \vdots \\
V_{(m+1)|m|}^{(Q)} & V_{(m+3)|m|}^{(Q)} & \ldots & V_{(N-1)|m|}^{(Q)}
\end{array}\right]
$$

with

$$
V_{n|m|}^{(q)}=-\sqrt{\frac{(2 n+1)\left(n^{2}-m^{2}\right)}{(2 n-1)}} j_{n}\left(k R_{q}\right) \mathcal{P}_{(n-1)|m|}(0)
$$

for the case with both $N$ and $m$ are either odd or even [otherwise replace $N-1$ in Eq. (28) by $N$ ].

We can estimate the odd harmonic coefficients from Eq. (26), provided $\boldsymbol{V}_{m}(k)$ is non-singular, as

$$
\boldsymbol{C}_{m}^{\mathrm{odd}}(k)=\boldsymbol{V}_{m}^{\dagger}(k) \alpha_{m}^{(\mathrm{d})}(k)
$$

where $\boldsymbol{V}_{m}^{\dagger}=\left(\boldsymbol{V}_{m}^{T} \boldsymbol{V}_{m}\right)^{-1} \boldsymbol{V}_{m}^{T}$ is the pseudo inverse of $\boldsymbol{V}_{m}$.
Thus the complete set of soundfield coefficients can be derived through solving for the even and odd harmonics coefficients separately using the signal received from omnidirectional microphones [Eq. (19)] and differential microphones [Eq. (26)], respectively.

\section{Cardioid or general first order microphone arrays}

Alternatively, the even and odd harmonic coefficients may be calculated together in one matrix operation. This method is especially suitable for planar arrays that utilize cardioid microphones (or general first order) instead of differential microphones. According to Eq. (14), a first order (e.g., cardioid) microphone placed on the $x$-y plane picks up both the even and odd components of the soundfield. For a set of finite radii circular arrays of first order microphones placed on the $x$ - $y$ plane, we can write a matrix equation using Eq. (14) and following similar steps as in Secs. III A 1 and III A 2 ,

$$
\alpha_{m}^{(\mathrm{f})}(k)=\beta \boldsymbol{U}_{m}(k) \boldsymbol{C}_{m}^{\mathrm{even}}(k)+(1-\beta) \boldsymbol{V}_{m}(k) \boldsymbol{C}_{m}^{\mathrm{odd}}(k)
$$

where $\alpha_{m}^{(\mathrm{f})}(k)=\left[\alpha_{m}^{(\mathrm{f})}\left(R_{1}, k\right), \alpha_{m}^{(\mathrm{f})}\left(R_{2}, k\right), \ldots, \alpha_{m}^{(\mathrm{f})}\left(R_{Q}, k\right)\right]^{T}$ with 


$$
\alpha_{m}^{(\mathrm{f})}\left(R_{q}, k\right) \triangleq \int_{0}^{2 \pi} P_{c}\left(R_{q}, \frac{\pi}{2}, \phi, k\right) E_{m}(-\phi) d \phi,
$$

and $\boldsymbol{C}_{m}^{\text {even }}(k), \boldsymbol{U}_{m}(k), \boldsymbol{C}_{m}^{\text {odd }}(k)$ and $\boldsymbol{V}_{m}(k)$ are given by Eqs. (20), (21), (27), and (28), respectively.

If we have both omni-directional and first order circular arrays of microphones, then we can combine Eqs. (19) and (31) to obtain

$$
\left[\begin{array}{c}
\alpha_{m}(k) \\
\alpha_{m}^{(\mathrm{f})}(k)
\end{array}\right]=\left[\begin{array}{cc}
\boldsymbol{U}_{m}(k) & 0 \\
\beta \boldsymbol{U}_{m}(k) & (1-\beta) \boldsymbol{V}_{m}(k)
\end{array}\right]\left[\begin{array}{c}
\boldsymbol{C}_{m}^{\text {even }}(k) \\
\boldsymbol{C}_{m}^{\text {odd }}(k)
\end{array}\right] .
$$

Equation (33) can be solved to calculate both the even and odd harmonics coefficients given by $\boldsymbol{C}_{m}^{\text {even }}(k)$ and $\boldsymbol{C}_{m}^{\text {odd }}(k)$.

\section{B. Discrete sensor placement: Sampling of continuous aperture}

In Sec. III A 3, we assumed that the pressure $P\left(R_{q}, \pi / 2, \phi, k\right)$, pressure gradient $P_{\theta}\left(R_{q}, \pi / 2, \phi, k\right)$ and the first order microphone output $P_{c}\left(R_{q}, \pi / 2, \phi, k\right)$ are readily available over a continuous circular aperture in Eqs. (17), (24), and (32), respectively. However, in practice, we only have a finite set of microphones and hence a discrete set of samples on the circular aperture. Thus for an equally spaced microphone arrays, we approximate the integration in Eqs. (17), (24), and (32) by summations,

$$
\begin{aligned}
& \alpha_{m}\left(R_{q}, k\right) \approx \frac{2 \pi}{N_{q}} \sum_{s=1}^{N_{q}} P\left(R_{q}, \pi / 2, \phi_{s}, k\right) E_{m}\left(-\phi_{s}\right), \\
& \alpha_{m}^{(\mathrm{d})}\left(R_{q}, k\right) \approx \frac{2 \pi}{N_{q}} \sum_{s=1}^{N_{q}} P_{\theta}\left(R_{q}, \frac{\pi}{2}, \phi_{s}, k\right) E_{m}\left(-\phi_{s}\right), \\
& \alpha_{m}^{(\mathrm{f})}\left(R_{q}, k\right) \approx \frac{2 \pi}{N_{q}} \sum_{s=1}^{N_{q}} P_{c}\left(R_{q}, \frac{\pi}{2}, \phi_{s}, k\right) E_{m}\left(-\phi_{s}\right),
\end{aligned}
$$

where $N_{q}$ are the number of microphones placed in a circle and $\phi_{s}$ denotes the azimuth angle of the location of the $s$ th microphone.

\section{Number of sensors per circle}

Due to the spatial sampling of the soundfield, one can only extract a limited number of harmonic orders by each array. To sample a set of circular harmonics of maximum order $N$, the number of microphones required is given by $n_{\text {mic }} \geq 2 N+1$. Due to inherent properties of the spherical Bessel functions, the circular harmonics that exists within a certain radius is order limited. A rule of thumb for deciding the maximum active harmonic order is given by $N \leq\lceil e k r / 2\rceil$, where $k$ is the wave number and $r$ is the radius of the region of interest. ${ }^{27}$ The exact amount of microphones to be used for each circular array thus depends on the radius of the array as well as the target frequency band.

The truncation of spherical harmonics leads to errors, which will be discussed in Sec. IV. The "rule of thumb" $N$ $\leq\lceil e k r / 2\rceil$ gives a sufficiently high precision for most applications. ${ }^{27}$ For applications that require less accuracy, an alternative truncation number is given by $N \leq\lceil k R\rceil .{ }^{25}$ The former rule is used in this paper for higher accuracy.

Because the number of microphones on each circular array is directly linked to the wave number $k$, which can then be translated into the wavelength $\lambda$, the number of microphones needed can be easily derived from the target frequency of the application as

$$
\begin{aligned}
n_{\text {mic }} & =2 N+1=2\left\lceil\frac{e k R}{2}\right\rceil+1=2\left\lceil\frac{e \pi R}{\lambda}\right\rceil+1 \\
& =2\left\lceil\frac{e \pi f R}{c}\right\rceil+1
\end{aligned}
$$

where $c$ is the speed of wave propagation, for audio applications, $c=340 \mathrm{~m} / \mathrm{s}$. Thus one can directly calculate the number of sampling points (microphones) for a given array radius and a target frequency band. For example, a circular array of $0.4 \mathrm{~m}$ radius, designed for audio signals up to $1500 \mathrm{~Hz}$, would need 33 microphones.

\section{Configuration(s)}

The array system can be configured to have multiple circular microphone arrays placed on a plane with half of the arrays using omni-directional microphones, the other half using first order microphones placed perpendicular to the plane. The number of microphones on each array is decided by the target wave number and the radius of the array, therefore smaller arrays may have a lower amount of microphones. Figure 1 illustrates such a configuration.

An alternative configuration is to use closely placed omni-directional microphone pairs to realize differential microphones. In this way, each microphone pair is used in two different ways: the two microphone output signals are differentiated to create the bi-directional pick up pattern, which is used for calculation of odd numbered coefficients; in the mean time, one of the two microphone outputs is used to calculate the even-numbered coefficients. Figure 2 shows an example of such array arrangement.

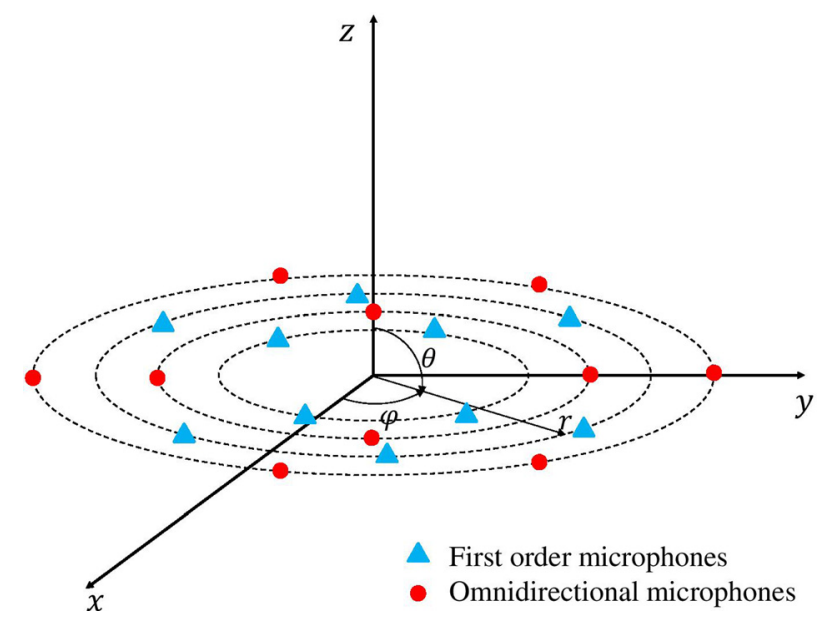

FIG. 1. (Color online) Example of omnidirectional (dot) and first order (triangle) microphone arrangement on a 2D plane for 3D soundfield analysis. 


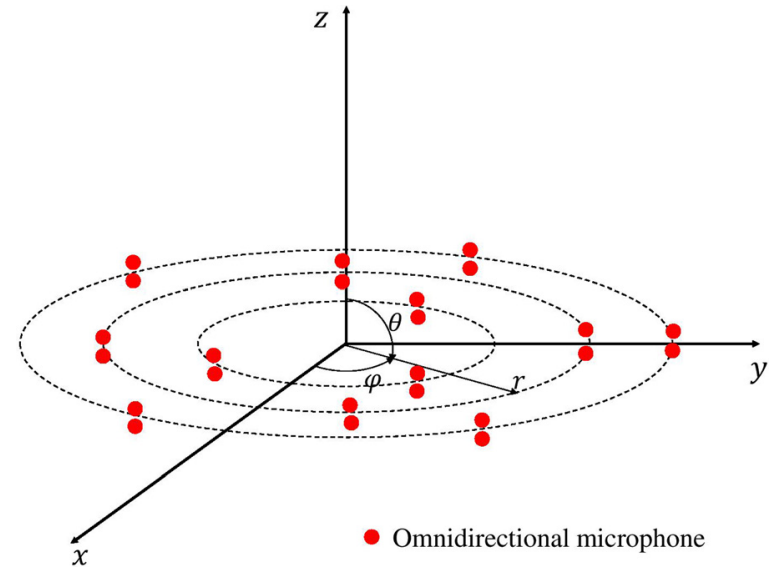

FIG. 2. (Color online) Example of omni-directional microphone pair arrangement on a $2 \mathrm{D}$ plane for $3 \mathrm{D}$ soundfield analysis.

The two microphone array configurations require the same number of microphones for the same design target, although the second option uses half the number of circular arrays. However, it should be noted that the distance between the two microphones in each microphone pair should be small compared to the array radius, so as to best approximate $P_{\theta}(r, \theta, \phi, k)$ in Eq. (5).

\section{Array design procedure}

A general guidance for designing the planar array is provided in this section. This procedure illustrates the basic steps in setting the parameters of the microphone array.

Step 1: Determine the desired frequency band and the radius $R$ of the region of interest.

Step 2: Calculate the maximum order of the soundfield using $N=\lceil e k R / 2\rceil$.

Step 3: Based on the maximum order $N$, decide the number of circular arrays to be implemented. For first order microphone configuration, at least $N_{\text {omni }}=\lceil N / 2\rceil$ omnidirectional sensor arrays and $N_{\text {first }}=N-N_{\text {omni }}$ first order arrays are needed. For differential microphone configuration, no less than $N_{\text {diff }}=\lceil N / 2\rceil$ arrays of microphone pairs are required.

Step 4: Determine the radius of each circular array. Choose the radius such that the spherical Bessel zeros for the target frequency band are avoided. Ensure that the radii of the circular arrays have a good diversity.

Step 5: For each circular array, decide the maximum spherical harmonic order $N_{i}$ and estimate the number of microphones to be placed on the array based on $n_{\text {mic }}=2 N_{i}+1$.

After settling on a design, the parameters for soundfield calculation can then be set based on the dimensions of the array.

\section{Comments}

We make the following comments and observations with the proposed array:
(1) The even spherical harmonics are symmetric about the $z=0(x-y)$ plane, while the odd modes are not. A planar microphone array comprising only omnidirectional microphones cannot distinguish the waves that are impinging from either sides of the plane. This fact explains that why this type of array is not capable of detecting the full 3D soundfield.

(2) First order cardioid microphones that are placed perpendicular to the array plane can pick up a combination of even and odd mode harmonics but are unable to separate the two components. However, if the even mode harmonic coefficients are known (which can be provided by an omnidirectional microphone array), then it becomes easy to solve for the remaining odd mode coefficients. Thus a hybrid array of both omnidirectional and first order microphones is crucial for detecting full 3D soundfield using a planar array aperture.

(3) The zeros in the spherical Bessel functions cause certain spherical harmonics to be "invisible" at some radius and frequency; this limits an array's wide band capabilities. The proposed array aperture samples the soundfield at multiple radii, thus improving the array's redundancy against zero points in the spherical Bessel functions. However, the user should carefully design the array such that at each frequency, a sufficient number of circular arrays are unaffected by the Bessel zeros and are available for calculating the coefficients. In general, a properly designed planar array can avoid the Bessel zero problem for all frequencies, and thus having wideband capabilities, this is shown in Sec. V using a hypothetical design example.

(4) Although the proposed array has a planar geometry, the free space assumption still applies to our array system, which requires that no sound source or scatterer should exist within the spherical region enveloping the planar array. For this reason, the array cannot be directly placed on walls or tables to capture the surrounding sound. However, a work-around to this problem is to place an appropriate sound absorbing material between the rigid surface (wall, table) and the planar array, which eliminates all reflections from the surface, thus the setup no longer violates the free-space assumption. Furthermore, if the reflection characteristics of the surface is known, it is possible to compensate for the reflection in the calculation. However, this is beyond the scope of this paper, and we will investigate this in a future work.

\section{ERROR ANALYSIS}

In this section, we discuss two primary sources of error, and the impact they have on the acquisition accuracy of different soundfield coefficients.

\section{A. Differential microphone approximation}

As was mentioned in Sec. III, a differential microphone can be realized using a pair of closely placed omni-directional microphones. However, this implementation only approximates the ideal velocity sensor, using the approximation 


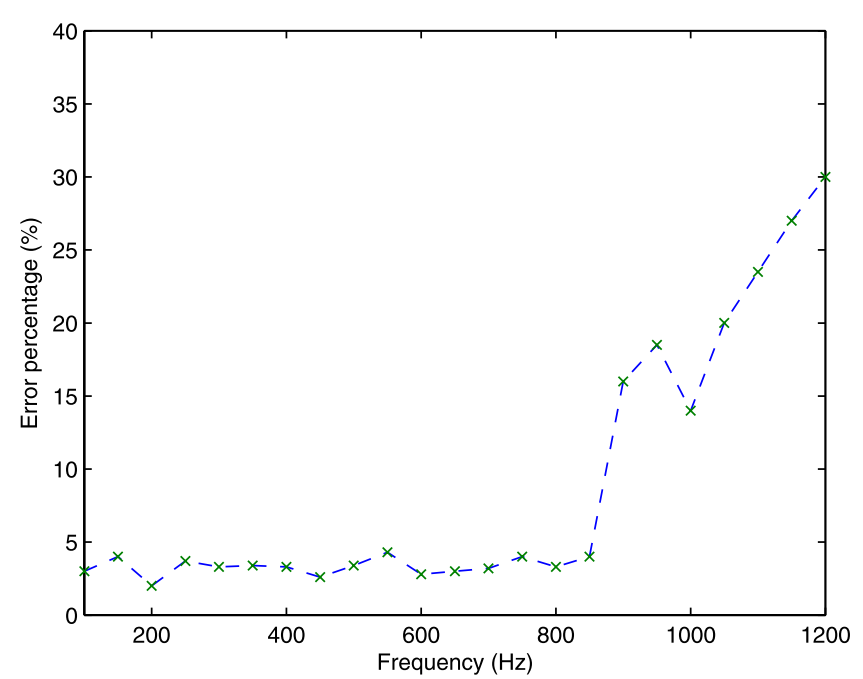

FIG. 3. (Color online) Reproduction error percentage for a point source of frequencies $150-1150 \mathrm{~Hz}$, located at $\left(1.6 \mathrm{~m}, 60^{\circ}, 90^{\circ}\right)$.

$$
\frac{P(\mathbf{x}+d \mathbf{x})-P(\mathbf{x})}{d \mathbf{x}} \approx \frac{\partial P(\mathbf{x})}{\partial \mathbf{x}}=V(\mathbf{x})
$$

By choosing sufficiently small value of $d \mathbf{x}$, the error of the approximation can be minimized. However, due to implementation constraints such as physical dimension of the microphone units, a very good approximation of Eq. (38) may not be achievable. We recommend choosing $d \mathbf{x} \approx 0.1 / k_{\max }$, where $k_{\max }$ is the wave number corresponding to the maximum operating frequency of the microphone array, so as to minimize the error due to the approximation.

Because this approximation only exists for the sampling of the odd coefficients, the accuracy of the calculated odd coefficients is expected to be slightly worse than that of the even coefficients when the differential microphone approximation is used to implement the array. This phenomenon is observed in the hypothetical design example.

\section{B. Spatial sampling and spatial aliasing}

One major source of error in the proposed array system is spatial sampling. By comparing Eq. (17) and its discrete approximation, Eq. (35), the error on each harmonic mode due to spatial sampling can be defined as

$$
\begin{aligned}
\Delta E_{\text {mode }} \triangleq & \int_{0}^{2 \pi} P(r, \theta, \phi, k) E_{m}(-\phi) d \phi \\
& -\frac{2 \pi}{n_{\text {mic }}} \sum_{u=1}^{n_{\text {mic }}} P(r, \theta(u), \phi(u), k) E_{m}(-\phi) .
\end{aligned}
$$

The same approximation error can be defined for Eqs. (24) and (32). Generally speaking, this error is small as long as the Nyquist sampling criteria is met; however, using extra microphones on each circular array can help to improve the accuracy of the system.

The truncation of spherical harmonic modes mentioned in Sec. III also leads to errors as the energy of the truncated higher order harmonics are aliased into the observed harmonics during calculation. The truncation error can be expressed as
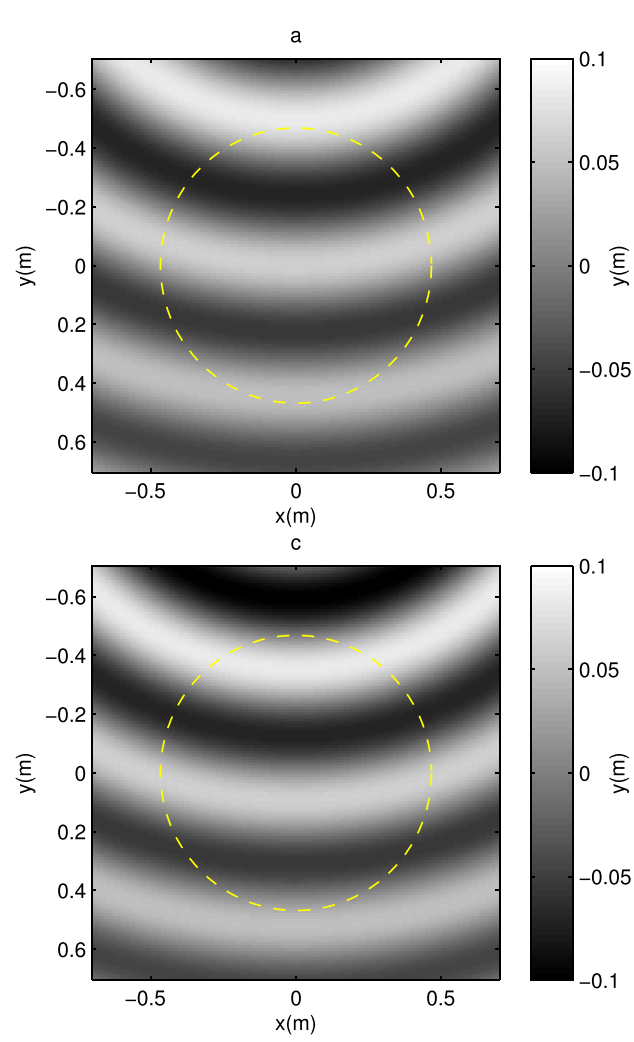

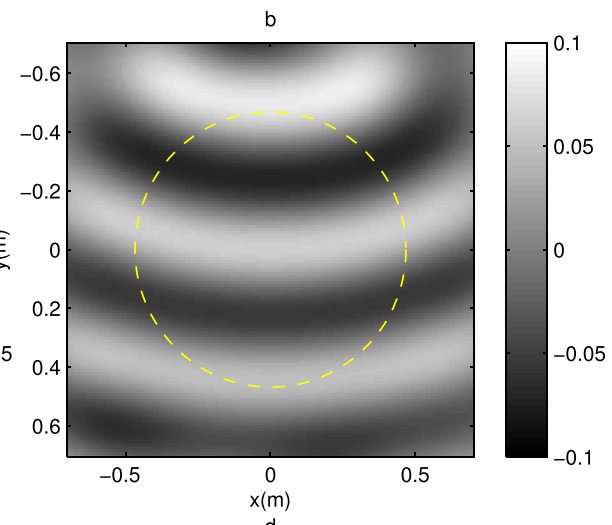

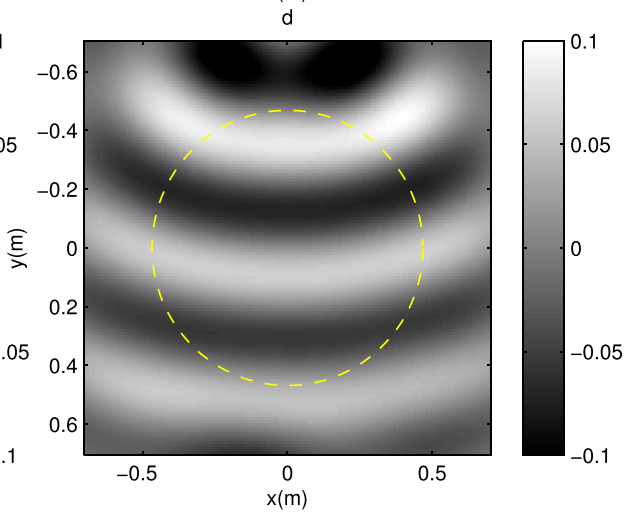

FIG. 4. (Color online) Actual [(a), (c)] and recorded $[(b),(d)]$ soundfield due to a $850 \mathrm{~Hz}$ point source located at $\theta=45^{\circ}, R=1.6 \mathrm{~m}$, reconstructed at $z=0[(\mathrm{a}),(\mathrm{b})]$ and $z=0.2 \mathrm{~m}[(\mathrm{c}),(\mathrm{d})]$ plane. 


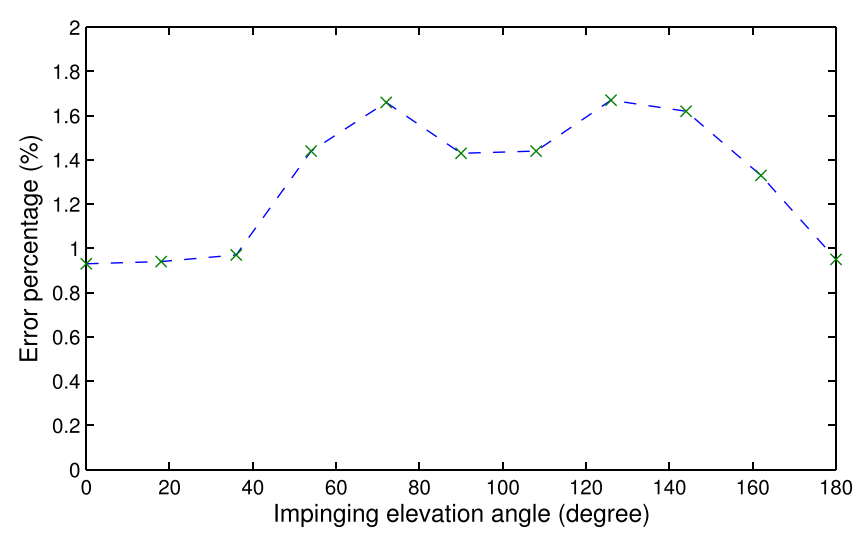

FIG. 5. (Color online) Reproduction error percentage for a plane wave source at $850 \mathrm{~Hz}$, moving from $\theta=0$ to $\theta=180^{\circ}$.

$$
\begin{aligned}
\Delta E_{\text {trunc }} \triangleq & \sum_{n=|m|}^{\infty} C_{n m} j_{n}(k r) \mathcal{P}_{n|m|}(0) \\
& -\sum_{n=|m|}^{N} C_{n m} j_{n}(k r) \mathcal{P}_{n|m|}(0) \\
= & \sum_{n=N+1}^{\infty} C_{n m} j_{n}(k r) \mathcal{P}_{n|m|}(0) .
\end{aligned}
$$

Using the rule of thumb given in Ref. 27, the error is in the order of $1 \%$. It should be noted that the truncation error will only be aliased into coefficients of the highest order due to inherent properties of the spherical Bessel functions.

Due to the structure of the proposed design example and the nature of the spherical Bessel functions, the lower order spherical harmonic modes are sampled by multiple circular arrays, whereas the highest order ones are only visible to one or two circular arrays. As a result, when solving for the soundfield coefficients using Eqs. (19) and (26), the lower order coefficients are less affected by the approximation and aliasing errors than the higher order coefficients. This trend is shown in Fig. 6.

\section{DESIGN EXAMPLES}

In this section, we describe (i) a hypothetical design example and (ii) an actual implementation of the proposed array. The purpose of the hypothetical example is to illustrate the procedures to design an array and to theoretically evaluate the array's capabilities. Then the implemented array is used to validate the technique through lab experiments.

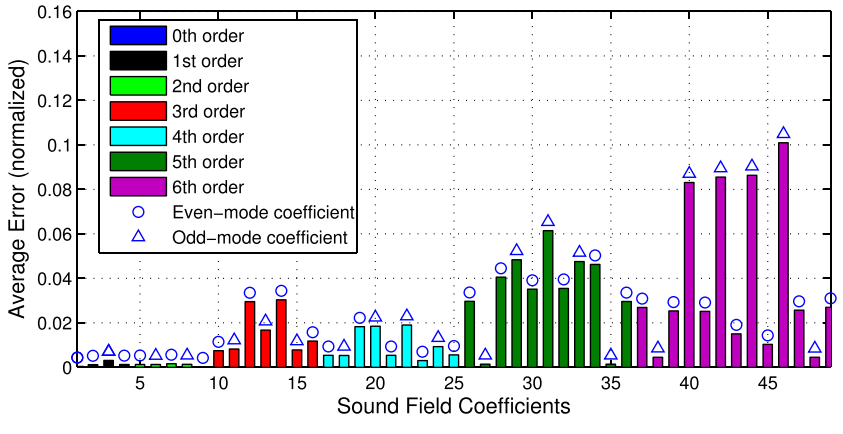

FIG. 6. (Color online) Average coefficient error due to a $500 \mathrm{~Hz}$ plane wave impinging from different elevation angles.

\section{A. Hypothetical design example}

We consider the case of recording the soundfield in a spherical region with a diameter of approximately $1 \mathrm{~m}$, the target frequency band is $50-850 \mathrm{~Hz}$. This design example illustrates the use of pairs of omni-directional microphones to realize differential microphones in the array. We chose this array configuration because its accuracy is worse compared to the design using both omni-directional and first order microphones, due to the presence of differential pattern approximation error mentioned in Sec. IV The radius of the array is chosen to be $0.46 \mathrm{~m}$, which is close to the size of the region of interest. Thus for the maximum frequency of $850 \mathrm{~Hz}$ and a radius of $0.46 \mathrm{~m}$, the array can pick up soundfield harmonics up to the order

$$
N=\left\lceil\frac{e k r}{2}\right\rceil=10,
$$

which means that the outer ring of the array should have at least $2 N+1=21$ microphone pairs. Following this manner, we place a series of circular arrays of different radii inside the outer circle. Following the design procedure given in Sec. III, the radii of the rings are set to be $0.46,0.4,0.34$, $0.28,0.22,0.16$, and $0.1 \mathrm{~m}$. Thus the number of microphone pairs on each ring are $21,19,17,13,11,9$, and 7 , respectively.

To evaluate the performance of the proposed array system, we place a single point source of frequency $150-1150 \mathrm{~Hz}$ at $(R, \theta, \phi)=\left(1.6 \mathrm{~m}, 60^{\circ}, 90^{\circ}\right)$. We use the array to estimate the spherical harmonic coefficients and then reconstructed the soundfield. We compare the reconstructed soundfield to the original soundfield and calculate the overall reproduction error of the system. Figure 3 depicts the error for different frequencies. Note that the error is

\begin{tabular}{|c|c|c|c|c|c|c|c|c|c|c|c|}
\hline & $m=0$ & $m=1$ & $m=2$ & $m=3$ & $m=4$ & $m=5$ & $m=6$ & $m=7$ & $m=8$ & $m=9$ & $m=10$ \\
\hline $100 \mathrm{~Hz}$ & 5.76 & 1.00 & 1.00 & I & / & / & / & / & / & / & / \\
\hline $200 \mathrm{~Hz}$ & 13.25 & 4.57 & 1.00 & 1.00 & / & / & / & / & / & / & / \\
\hline $400 \mathrm{~Hz}$ & 46.30 & 19.38 & 15.97 & 6.33 & 1.00 & 1.00 & / & I & / & / & / \\
\hline $800 \mathrm{~Hz}$ & 181.35 & 21.80 & 110.99 & 13.24 & 54.40 & 41.80 & 10.88 & 4.20 & 7.88 & 1.00 & 1.00 \\
\hline
\end{tabular}
small when the frequency is below $850 \mathrm{~Hz}$, which is the desired maximum frequency for the array. Beyond the upper

TABLE I. Condition number of matrix $\boldsymbol{U}_{m}$ of the hypothetical design example for frequencies $100,200,400$, and $800 \mathrm{~Hz}$. 


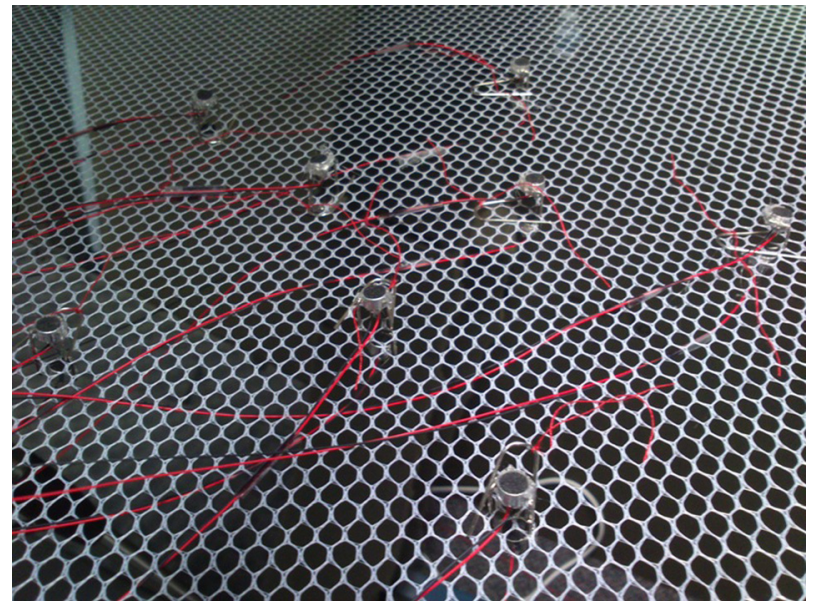

FIG. 7. (Color online) Implemented planar microphone array, using omnidirectional microphone pairs.

frequency, the error percentage increases dramatically. The reason is that as the frequency increases, the order of active spherical harmonics also grows. At frequencies above $850 \mathrm{~Hz}$, the number of microphones needed to estimate the higher frequency components are greater than the number of the microphones on the array, thus causing aliasing. Also the total number of coefficients for each mode $m$ exceeds the number of circular arrays available, as a result, the matrix inversion problems shown in Eqs. (19) and (26) become under-determined, resulting in significant errors.

We plot the original and reconstructed (using captured spherical harmonic coefficients) soundfields in Fig. 4, where plots (a) and (c) are the actual soundfield at planes $z=0$ and $z=0.2 \mathrm{~m}$, (b) and (d) are the recorded and reconstructed soundfield at these two planes, respectively. We observe that the captured soundfield over the region of interest in both planes are similar to the actual soundfield in the same area.

To evaluate the array performance for different impinging angles, we move a plane wave source at frequency $850 \mathrm{~Hz}$ over different elevation angles over $\left[0,180^{\circ}\right]$ and the corresponding reproduction error is given in Fig. 5. As seen from Fig. 5, the error is less than 1.8\% over all elevation angles. Due to the symmetry of the array over the azimuth angles, the performance are almost constant over different azimuth angles.
To examine the array accuracy in terms of soundfield coefficients, we move a plane wave source at frequency $500 \mathrm{~Hz}$ over different elevation angles in the range of $\left[0,180^{\circ}\right]$ and calculate the average error for each coefficient, where the theoretical coefficient response to a plane wave impinging from $(\vartheta, \varphi)$ is given by ${ }^{23}$

$$
\alpha_{n m}=\sqrt{4 \pi} i^{n} Y_{n m}(\vartheta, \varphi)^{*} .
$$

Figure 6 plots the normalized average error for each coefficient. It can be observed that the lower order coefficients are more accurately measured compared to the higher order ones; also, the even mode coefficients are more accurate compared to the odd mode coefficients.

Table I shows the condition number of the matrix $\boldsymbol{U}_{m}$ of the designed array for various frequencies. Due to the separation of the even and odd mode harmonic coefficients, the coefficients $C_{N, \pm N}, C_{N, \pm(N-1)}$ and $C_{N-1, \pm(N-1)}$ are solved uniquely, therefore the matrices $\boldsymbol{U}_{N}$ and $\boldsymbol{U}_{N-1}$ are in fact vectors whose eigenvalues equal to 1 . The size of $\boldsymbol{U}_{m}$ grows as the frequency increases, and the condition number for lower modes increase correspondingly. The design example consists of the minimum number of circular arrays. We expect the condition numbers to be lower should additional circular arrays be used in the system. Also, for high order systems $(N \geq 5)$, regularization should be applied when inverting the matrix $\boldsymbol{U}_{m}$.

In general, we can see from the simulations that the design example offers good accuracy, with its error in the order of $1 \%$. This is comparable to the performance of spherical microphone arrays ${ }^{7}$ and other previously proposed array configurations such as the multiple circular microphone array $^{21}$ and the double sided cone $\operatorname{array}^{20}$ of the same order, assuming that a similar number of microphones have been used in each array configuration.

\section{B. Array implementation}

To experimentally test the proposed array design and the associated algorithms, we built a physical array of omnidirectional microphones (see Fig. 7). Due to hardware limitations, we only use 16 microphones to build the array. Therefore the array is designed to detect up to the second order soundfield for up to $1000 \mathrm{~Hz}$ frequency. Based on the proposed design procedure, the system is built to have two
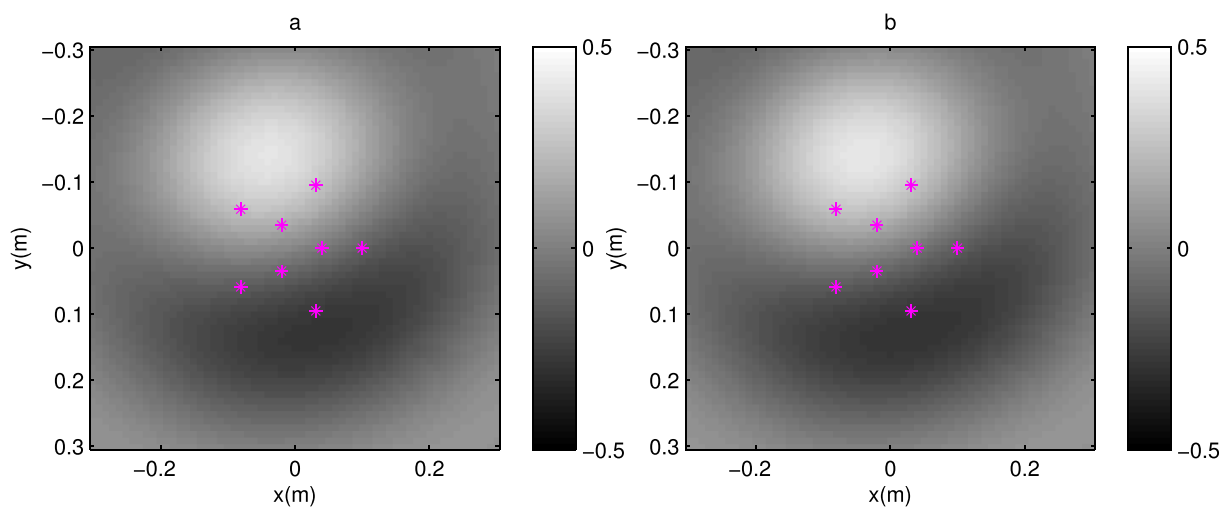

FIG. 8. (Color online) Comparison of (a) recorded and (b) simulated soundfield for a $850 \mathrm{~Hz}$ source at $(R, \theta, \phi)=\left(1.64 \mathrm{~m}, 45^{\circ}, 100^{\circ}\right)$, reconstructed at the $z=0.05 \mathrm{~m}$ plane. 
TABLE II. Soundfield coefficient comparison between simulation and experimental results, the soundfields are due to a point source located at $(R$, $\theta$, $\phi)=\left(1.64 \mathrm{~m}, 45^{\circ}, 100^{\circ}\right)$ and $\left(1.5 \mathrm{~m}, 90^{\circ}, 225^{\circ}\right)$, respectively.

\begin{tabular}{|c|c|c|c|c|c|c|c|c|c|c|}
\hline & Coefficient & $C_{00}$ & $C_{1(-1)}$ & $C_{10}$ & $C_{11}$ & $C_{2(-2)}$ & $C_{2(-1)}$ & $C_{20}$ & $C_{21}$ & $C_{22}$ \\
\hline \multirow[t]{4}{*}{ Recording 1} & Recorded & 1.5413 & 0.9608 & 0.9936 & 0.8351 & 0.9566 & 0.7312 & 1.0255 & 1.3735 & 0.5825 \\
\hline & Simulated & 1.1079 & 0.9569 & 1.1892 & 0.9626 & 0.8198 & 1.4140 & 0.5095 & 1.4513 & 0.6788 \\
\hline & Magnitude Error & 0.5142 & 0.0040 & 0.1645 & 0.1325 & 0.1668 & 0.4829 & 1.1026 & 0.0536 & 0.1418 \\
\hline & Phase Error & 0.0009 & 0.0226 & 0.1510 & 0.0888 & 0.4383 & 0.3350 & 0.0145 & 0.1566 & 0.3904 \\
\hline \multirow[t]{4}{*}{ Recording 2} & Recorded & 1.7838 & 1.5167 & 0.0968 & 1.5957 & 1.7211 & 0.1066 & 0.6772 & 0.1121 & 1.3902 \\
\hline & Simulated & 1.2380 & 1.4368 & 0 & 1.4137 & 1.6001 & 0 & 1.3127 & 0 & 1.8995 \\
\hline & Magnitude Error & 0.5457 & 0.0798 & / & 0.1820 & 0.1209 & / & -0.6355 & / & -0.5094 \\
\hline & Phase Error & -0.0515 & 0.1143 & / & -0.1372 & -0.1365 & / & -3.9421 & / & -0.4036 \\
\hline
\end{tabular}

co-centered circular arrays, the outer ring has the radius of $10 \mathrm{~cm}$, consisting of five omnidirectional microphone pairs, while the inner ring is $4 \mathrm{~cm}$ in radius, and consists of three microphone pairs.

Testing of the microphone array was conducted in our acoustic lab. A series of factors contribute to the errors in the test results. First of all, although most rigid surfaces in the lab are covered by acoustic foams to reduce reverberation, the acoustic foams are relatively thin and thus reverberations still exist. Second, the microphone capsules used have a sensitivity variation of approximately $6 \mathrm{~dB}$, and the calibration process could not guarantee high uniformity among all the microphone units. This factor has a significant impact on the performance of the differential microphone pairs. Furthermore, the position of each microphone unit has a deviation of $1-2 \mathrm{~mm}$, which also leads to errors in the acquired data.

In our experiment, the impinging soundfields are due to two loudspeakers that play $850 \mathrm{~Hz}$ sine waves. The loudspeakers were placed at $(R, \theta, \phi)=\left(1.64 \mathrm{~m}, 45^{\circ}, 100^{\circ}\right)$ and $\left(1.5 \mathrm{~m}, 90^{\circ}, 225^{\circ}\right)$, respectively. To evaluate the results of the experiments, the same loudspeaker-microphone array setup is simulated using MATLAB. Figure 8 plots the recorded soundfield (a) and the simulated soundfield (b) due to a point source located at $(R, \theta, \phi)=\left(1.64 \mathrm{~m}, 45^{\circ}, 100^{\circ}\right)$. It can be seen from the figure that the recorded soundfield is very similar to the simulated result.

Table II lists the spherical harmonic coefficients calculated from the recorded data as well as those acquired from the simulation results. It can be seen that although rather significant errors occur with some coefficients, the general patterns match very well. The microphone data used are raw recordings processed by microphone calibration data, which were acquired before assembling the array, therefore all the errors mentioned previously are present and have an impact on the recorded coefficients. Further calibration to the system, including microphone gain calibration, array geometry adjustments, and modification of algorithm parameters can be expected to greatly improve the accuracy of the system.

We would like to point out that our array system utilizes 16 microphones to capture second order soundfield, whereas in theory, the minimum number of microphones required to capture second order soundfield is nine. Therefore the proposed array system does not reduce the number of microphones required to sample the soundfield. The highlight of our proposed array structure is that it reduces the physical dimension of a higher order microphone array system without compromising its functionality.

\section{CONCLUSION}

This paper introduces a method of measuring complete $3 \mathrm{D}$ soundfield information on a $2 \mathrm{D}$ plane through the combined use of omnidirectional microphones and first order microphones. We provide two options for planar microphone array implementation based on the proposed soundfield measuring method. Both array configurations consist of multiple co-centered circular arrays with one option using both omni-directional microphones and first order microphones, while the other option using omni-directional microphones only. The associated algorithms to calculate soundfield coefficients are also given in the paper. We show in the simulation example that the proposed 2D microphone array system has good accuracy within its designed operating frequency band, and both even and odd soundfield coefficients can be accurately calculated. We also built an experimental planar microphone array to further validate the proposed theory.

${ }^{1}$ E. Tiana-Roig, F. Jacobsen, and E. Fernandez-Grande, "Beamforming with a circular array of microphones mounted on a rigid sphere (L)," J. Acoust. Soc. Am. 130(3), 1095-1098 (2011).

${ }^{2}$ S. Yan, H. Sun, U. Svensson, X. Ma, and J. Hovem, "Optimal modal beamforming for spherical microphone arrays," IEEE Trans. Audio Speech, Language Process. 19(2), 361-371 (2011).

${ }^{3}$ C. C. Lai, S. Nordholm, and Y. H. Leung, "Design of steerable spherical broadband beamformers with flexible sensor configurations," IEEE Trans. Audio Speech Language Process. 21(2), 427-438 (2013).

${ }^{4}$ H. Sun, E. Mabande, K. Kowalczyk, and W. Kellermann, "Localization of distinct reflections in rooms using spherical microphone array eigenbeam processing,” J. Acoust. Soc. Am. 131(4), 2828-2840 (2012).

${ }^{5}$ D. Khaykin and B. Rafaely, "Acoustic analysis by spherical microphone array processing of room impulse responses," J. Acoust. Soc. Am. 132(1), 261-270 (2012).

${ }^{6}$ T. D. Abhayapala and D. B. Ward, "Theory and design of high order sound field microphones using spherical microphone array," in 2002 IEEE International Conference on Acoustics, Speech, and Signal Processing (ICASSP), Vol. 2, pp. II-1949-II-1952.

${ }^{7} \mathrm{~B}$. Rafaely, "Analysis and design of spherical microphone arrays," IEEE Trans. Speech Audio Process. 13(1), 135-143 (2005).

${ }^{8}$ H. Teutsch and W. Kellermann, "Detection and localization of multiple wideband acoustic sources based on wavefield decomposition using spherical apertures," in IEEE International Conference on Acoustics, Speech and Signal Processing, 2008, ICASSP, pp. 5276-5279.

${ }^{9}$ D. Khaykin and B. Rafaely, "Coherent signals direction-of-arrival estimation using a spherical microphone array: Frequency smoothing approach," in IEEE Workshop on Applications of Signal Processing to Audio and Acoustics, 2009, WASPAA'09, pp. 221-224. 
${ }^{10}$ Y. Peled and B. Rafaely, "Method for dereverberation and noise reduction using spherical microphone arrays," in 2010 IEEE International Conference on Acoustics Speech and Signal Processing (ICASSP), pp. 113-116.

${ }^{11}$ D. Jarrett and E. A. P. Habets, "On the noise reduction performance of a spherical harmonic domain tradeoff beamformer," IEEE Signal Process. Lett. 19(11), 773-776 (2012).

${ }^{12}$ E. de Witte, H. Griffiths, and P. Brennan, "Phase mode processing for spherical antenna arrays," Electron. Lett. 39(20), 1430-1431 (2003).

${ }^{13}$ E. Mabande, K. Kowalczyk, H. Sun, and W. Kellermann, "Room geometry inference based on spherical microphone array eigenbeam processing," J. Acoust. Soc. Am. 134(4), 2773-2789 (2013).

${ }^{14}$ F. Jacobsen, G. Moreno-Pescador, E. Fernandez-Grande, and J. Hald, "Near field acoustic holography with microphones on a rigid sphere (L)," J. Acoust. Soc. Am. 129(6), 3461-3464 (2011).

${ }^{15}$ I. Balmages and B. Rafaely, "Open-sphere designs for spherical microphone arrays," IEEE Trans. Audio, Speech, Language Process. 15(2), 727-732 (2007).

${ }^{16}$ T. D. Abhayapala and M. C. T. Chan, "Limitation and errior analysis of spherical microphone arrays," in 14th International Congress on Sound and Vibration (ICSV14), Cairns, Australia (July 2007).

${ }^{17}$ C. Jin, A. Parthy, and A. Van Schaik, "Optimisation of co-centred rigid and open spherical microphone arrays," in Proceedings of the 120th Audio Engineering Society Convention, Paris, France (May 2006), 6 pp.

${ }^{18}$ B. Rafaely, "The spherical-shell microphone array," IEEE Trans. Audio, Speech, Language Process. 16(4), 740-747 (2008).
${ }^{19} \mathrm{Z}$. Li and R. Duraiswami, "Flexible and optimal design of spherical microphone arrays for beamforming," IEEE Trans. Audio, Speech, Language Process. 15(2), 702-714 (2007).

${ }^{20}$ A. Gupta and T. D. Abhayapala, "Double sided cone array for spherical harmonic analysis of wavefields," in IEEE International Conference on Acoustics Speech and Signal Processing (ICASSP) (March 2010), pp. 77-80.

${ }^{21}$ T. D. Abhayapala and A. Gupta, "Spherical harmonic analysis of wavefields using multiple circular sensor arrays," IEEE Trans. Audio, Speech, Language Process. 18(6), 1655-1666 (2010).

${ }^{22}$ J. Meyer and G. Elko, "Spherical harmonic modal beamforming for an augmented circular microphone array," in IEEE International Conference on Acoustics, Speech and Signal Processing, 2008, ICASSP, pp. 5280-5283.

${ }^{23}$ H. Teutsch, Modal Array Signal Processing: Principles and Applications of Acoustic Wavefield Decomposition (Springer, New York, 2007), Chap. 3, pp. 53-54.

${ }^{24}$ R. R. Achim Kuntz, "Cardioid pattern optimization for a virtual circular microphone array," in 2009 Proceedings of the EAA Symposium on Auralizatio, Espoo, Finland, 2009.

${ }^{25}$ D. B. Ward and T. D. Abhayapala, "Reproduction of a plane-wave sound field using an array of loudspeakers," IEEE Trans. Speech Audio Process. 9, 697-707 (2001).

${ }^{26}$ I. Gradshteyn and I. Ryzhik, Table of Integrals, Series, and Products (Academic, New York, 2000), p. 955.

${ }^{27}$ R. A. Kennedy, P. Sadeghi, T. D. Abhayapala, and H. M. Jones, "Intrinsic limits of dimensionality and richness in random multipath fields," IEEE Trans. Signal Process. 55(6), 2542-2556 (2007). 